\title{
Operando Transmission X-ray Microscopy Studies on Li-Ion Batteries
}

\author{
Johanna Nelson Weker ${ }^{1,}$ Joy C. Andrews ${ }^{1}$, Yi Cui ${ }^{2}$, William Chueh ${ }^{2}$, Michael F. Toney ${ }^{1}$ \\ ${ }^{1}$ Stanford Synchrotron Radiation Lightsource, SLAC National Accelerator Laboratory, Menlo Park, CA \\ ${ }^{2}$ Stanford University, Stanford, CA
}

A fundamental component in the global move towards clean, renewable energy is the electrification of the automobile. Current battery technology limits electric vehicles to a short travel range, slow recharge, and costly price tag. $\mathrm{Li}$-ion batteries promise the high specific capacity required to replace the internal combustion engine with a number of possible earth abundant electrode materials; however, setbacks such as capacity fading hinder the full capability of these rechargeable batteries.

Using the penetrative power of full-field hard X-ray transmission microscopy (TXM), we are able to track morphological $[1,2]$ and chemical changes of electrode material in real time during typical battery operation to better understand the electrochemical reactions and failure mechanisms (Figure 1). We will discuss recent results tracking the morphology evolution of anode materials in 2D (Figure 2) and 3D (Figure 3). With these techniques we show the first unambiguous demonstration of alloying anode materials fracturing into completely isolated pieces during cycling. Additionally, a strong size dependence for particle participation in the second electrochemical cycle has been discovered. This can account for up to a quarter of the capacity lost after the first cycle.

By combining X-ray absorption spectroscopy with high resolution TXM [3, 4], we have tracked electrochemical changes in micron and nanometer sized cathode materials during cycling. We will show dramatically inhomogenous charging and discharging within a $\sim 30 \mu \mathrm{m} \times 30 \mu \mathrm{m}$ field of view of a cathode comprised of agglomerates of nanoparticles. Moreover, a clear "memory" effect is seen in the switch from charge to discharge. Regions which are the last to charge are the regions which are first to discharge. Finally, substantial chemical and morphological changes are visible during deep discharges of $\mathrm{LiCoO}_{2}$, a standard cathode material (Figure 4). Additionally, a clear core/shell phenomenon is visible in these micron-sized particles.

[1.] Nelson, J., et al., Journal of American Chemical Society, 2012. 134(14): p. 6337-43.

[2.] Nelson, J., et al. Identifying and managing radiation damage during in situ transmission $\mathrm{x}$-ray microscopy of Li-ion batteries. 2013.

[3.] Meirer, F., et al., Journal of Synchrotron Radiation, 2011. 18(Pt 5): p. 773-81.

[4.] Boesenberg, U., et al., Chemistry f Materials, 2013. 25(9): p. 1664-1672. 

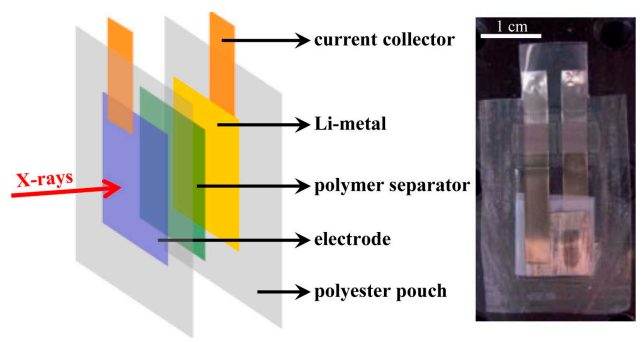

Figure 1. In situ $\mathrm{X}$-ray transparent pouch cell for multimodal X-ray characterization including TXM.

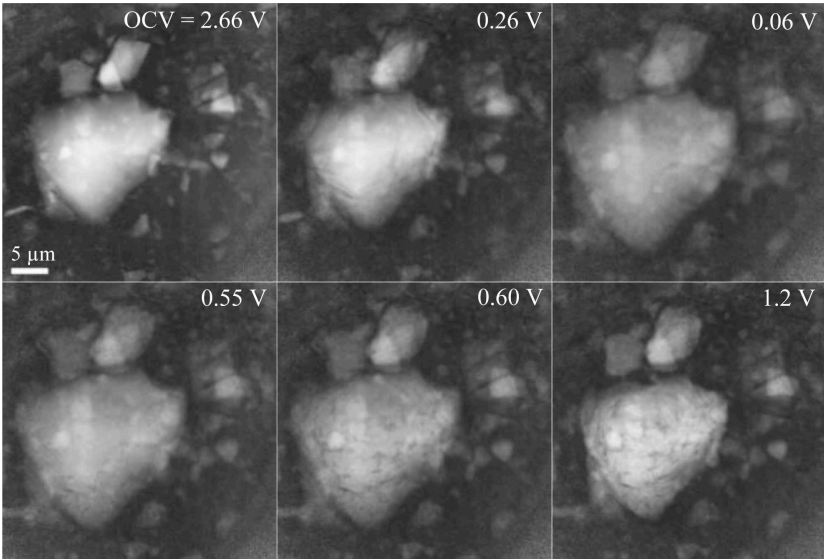

Figure 2. In situ TXM absorption snapshots of micron-size germanium particles during the first lithiation (top) and delithiation (bottom) cycle. The voltages at which the images were recorded are given in the top right corner of each panel.

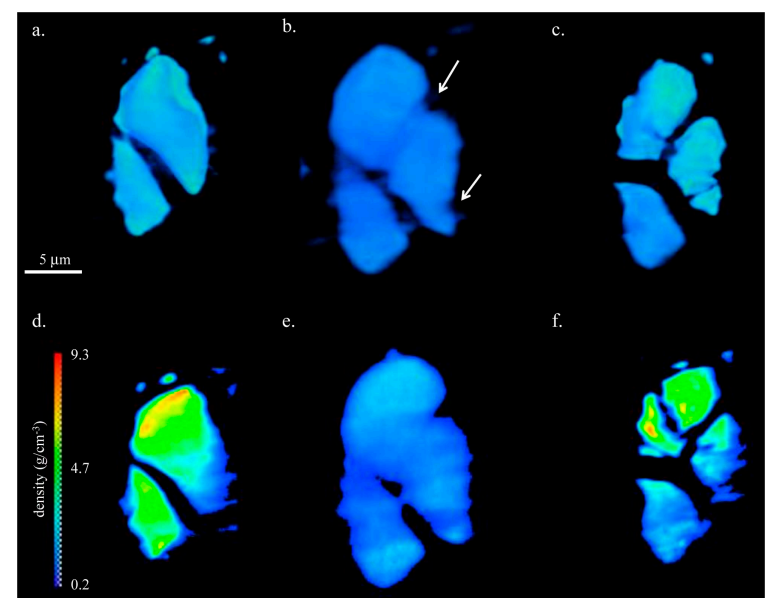

Figure 3. Volume renderings with corresponding cross-sections through the volume from in situ tomographic imaging of Ge particles $(\mathrm{a}, \mathrm{d})$ before cycling, $(\mathrm{b}, \mathrm{e})$ after the first lithiation cycle, and $(\mathrm{c}, \mathrm{f})$ after the first delithiation cycle.

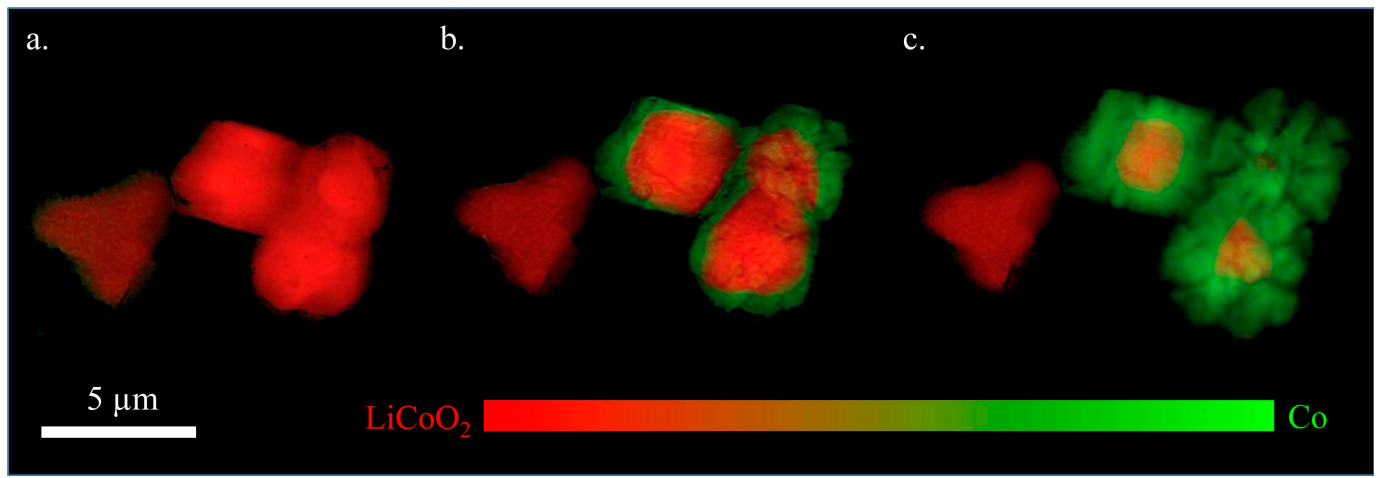

Figure 4. In situ chemically resolved TXM images of $\mathrm{LiCoO}_{2}$ particles before (a) and during (b, c) a deep battery discharge. Microns sized $\mathrm{LiCoO}_{2}$ particles pulverized into nanoparticles of Co. Images show the transformation evolving in the core/shell manner and the micron sized particles expanding and cracking. 